\title{
Distanciamiento físico óptimo, máscaras faciales y protección ocular para prevenir la transmisión de persona a persona del SARS-CoV-2
}

\author{
Optimal physical distance, face masks, and eye protection to prevent person-to-person transmission of SARS-CoV-2
}

\section{Comentado de:}

Chu DK et al. The Lancet. 2020;395(10242):1973-1987. PMID: $32497510^{1}$

\section{Objetivo}

Investigar los efectos del distanciamiento físico, las máscaras faciales y la protección ocular contra la transmisión del virus en entornos de atención médica y no médica (comunidad).

\section{Métodos de búsqueda}

Se realizaron búsquedas electrónicas (hasta el 26 de marzo de 2020) en MEDLINE(Ovid), PubMed, Embase, CINAHL, Cochrane Library, COVID-19 Open Research Dataset Challenge, la base de datos de investigación COVID-19 de la Organización Mundial de la Salud, Epistemonikos, el mapa sistemático vivo de la evidencia del Centro EPPI y registros de ensayos clínicos, además de revisar sitios web gubernamentales y de organizaciones relevantes, listas de referencias y revisiones sistemáticas relevantes. Adicionalmente, se realizaron búsquedas manuales en servidores de preimpresiones y centros de recursos sobre coronavirus de revistas científicas, al 3 de mayo de 2020 .

Se incluyeron estudios de cualquier diseño y en cualquier entorno que incluyera pacientes según criterios definidos por la OMS como caso confirmado o probable de COVID-19, Síndrome Agudo Respiratorio Severo (conocido como SARS, por sus iniciales en inglés) o Síndrome Respiratorio del Medio Oriente (MERS, por sus iniciales en inglés) y personas en contacto cercano con ellos, comparando distancias entre personas y pacientes infectados con COVID-19 de un metro o más, con o sin una máscara facial en el paciente, o con o sin máscara facial, protección para los ojos o ambas intervenciones en el individuo expuesto. No se aplicaron restricciones por idioma.

Criterios de selección, recolección y análisis de datos

La selección de los registros, la extracción de los datos y la evaluación del riesgo de sesgo se realizaron por duplicado. Se realizaron meta-análisis frecuentistas y bayesianos, y metaregresiones con modelo de efectos aleatorios. La confianza en la evidencia se valoró de acuerdo con los métodos Cochrane y el enfoque GRADE.

\section{Resultados principales}

Se incluyeron 172 estudios observacionales, realizados en 16 países y seis continentes, y 44 estudios comparativos en entornos de atención médica y no médica, con un total de 25.697 pacientes. Siete de estos estudios se centraron en COVID-19 (6.674 participantes), 26 en SARS (15.928) y 11 en MERS (3.095). No se identificaron ensayos aleatorizados de ninguna de las intervenciones de interés que abordaran directamente las poblaciones de estudio elegibles.

En la Tabla 1 se resumen las medidas de efecto de los desenlaces principales.

Tabla 1. Resumen GRADE de los hallazgos: probabilidades de infección o transmisión viral con las intervenciones evaluadas. Fuente: modificado de Chu DK et al. The Lancet. 2020;395(10242):1973-1987. Notas: ORa = odds ratio ajustado; $R R=$ riesgo relativo

\begin{tabular}{|c|c|c|c|c|c|}
\hline $\begin{array}{l}\text { Intervenciones } \\
\text { evaluadas }\end{array}$ & $\begin{array}{l}\text { Efecto relativo } \\
\text { (IC } 95 \%)\end{array}$ & $\begin{array}{l}\text { Riesgo Absoluto } \\
\text { anticipado (IC } 95 \%) \\
\text { en el grupo control }\end{array}$ & $\begin{array}{l}\text { Riesgo Absoluto } \\
\text { anticipado (IC } 95 \% \text { ) en } \\
\text { el grupo intervención }\end{array}$ & $\begin{array}{l}\text { Diferencia de riesgo } \\
\text { (IC } 95 \%)\end{array}$ & $\begin{array}{l}\text { Confianza en la } \\
\text { evidencia* }\end{array}$ \\
\hline $\begin{array}{l}\text { Distancia física } \geq 1 \\
\mathrm{~m} \text { vs. }<1 \mathrm{~m}\end{array}$ & $\begin{array}{c}\text { ORa } 0,18 \\
(0,09 \text { a } 0,38) \\
\text { RR } 0,30(0,20 \text { a } 0,44)\end{array}$ & $\begin{array}{c}\text { Distancia menor } \\
12,8 \%\end{array}$ & $\begin{array}{c}\text { Distancia mayor } 2,6 \% \\
(1,3 \text { a } 5,3)\end{array}$ & $\begin{array}{l}-10,2 \% \\
(-11,5 \mathrm{a}-7,5)\end{array}$ & $\begin{array}{c}\oplus \oplus \oplus \ominus \\
\text { MODERADA }\end{array}$ \\
\hline $\begin{array}{l}\text { Máscara facial vs. } \\
\text { sin máscara }\end{array}$ & $\begin{array}{c}\text { ORa } 0,15 \\
(0,07 \text { a } 0,34) ; \\
\text { RR } 0,34(0,26 \text { a } 0,45) \\
\end{array}$ & $\begin{array}{c}\text { Sin máscara } \\
17,4 \%\end{array}$ & $\begin{array}{l}\text { Con máscara } \\
3,1 \%(1,5 \text { a } 6,7)\end{array}$ & $\begin{array}{c}-14,3 \% \\
(-15,9 a-10,7)\end{array}$ & $\begin{array}{r}\oplus \oplus \ominus \ominus \\
\text { BAJA }\end{array}$ \\
\hline $\begin{array}{l}\text { Protección ocular } \\
\text { (careta, gafas) vs. } \\
\text { sin protección }\end{array}$ & RR $0,34(0,22$ a 0,52$)$ & $\begin{array}{c}\text { Sin protección ocular } \\
16,0 \%\end{array}$ & $\begin{array}{c}\text { Con protección ocular } \\
5,5 \%(3,6 \text { a } 8,5)\end{array}$ & $\begin{array}{l}-10,6 \% \\
(-12,5 \mathrm{a}-7,7)\end{array}$ & $\begin{array}{r}\oplus \oplus \ominus \ominus \\
\text { BAJA }\end{array}$ \\
\hline
\end{tabular}

\section{Clasificación GRADE}

Alta calidad: Estamos muy seguros de que el verdadero efecto se encuentra cerca de la estimación del efecto;

Calidad moderada: Tenemos una confianza moderada en la estimación del efecto: es probable que el efecto real esté próximo a la estimación del efecto, pero existe la posibilidad de que sea sustancialmente diferente;

Baja calidad: Nuestra confianza en la estimación del efecto es limitada: El verdadero efecto puede ser sustancialmente diferente de la estimación del efecto;

Muy baja calidad: Tenemos muy poca confianza en la estimación del efecto: Es probable que el efecto real sea sustancialmente diferente de la estimación del efecto. 


\section{Conclusiones}

Los resultados de esta revisión sistemática y meta-análisis respaldan el distanciamiento físico de un metro o más y proporcionan estimaciones cuantitativas para informar modelos de simulación y políticas de rastreo de contactos. Los datos también sugieren que el uso de máscaras faciales protegería a las personas (tanto trabajadores de la salud como el público en general) contra la infección por coronavirus, y la protección ocular podría conferir un beneficio adicional. Sin embargo, ninguno de estas intervenciones brindaron protección comple- ta contra la infección, y su papel óptimo podría necesitar una evaluación de riesgos y varias consideraciones contextuales.

Fuente de financiamiento/Conflicto de interés de los autores: El estudio fue financiado en parte por la OMS. Mark Loeb es investigador de un ensayo clínico en curso sobre máscaras médicas versus respiradores N95 para COVID-19 (NCT04296643). Todos los demás autores declararon no tener conflicto de interés.

\section{Comentario}

A la fecha de publicación de este resumen y comentario, el nuevo coronavirus SARS-CoV-2 ha infectado a más de 10 millones de personas en todo el mundo y causado más de 500.000 muertes $^{2}$. Sin intervenciones farmacológicas efectivas o vacunas disponibles en un futuro inminente, reducir la tasa de infección (es decir, aplanar la curva) es una prioridad, y la prevención de la infección es el mejor enfoque para lograr este objetivo.

Muchos países y regiones de todo el mundo iniciaron bloqueos de emergencia, con efectos sobre la salud, el bienestar, los negocios y otros aspectos de la vida cotidiana que afectan a las sociedades e individuos. Los consejos para reducir la transmisión de COVID-19 emitidos en el transcurso de la pandemia respecto al distanciamiento social fueron contradictorios, en base a información limitada. Además, se ha debatido sobre si la utilización de máscaras y cubiertas para los ojos podrían reducir la transmisión de COVID-19 en la población general, y cuál sería el uso óptimo de máscaras en entornos de atención médica ${ }^{3}$.

Esta revisión sistemática concluye que el distanciamiento físico de al menos un metro reduciría el riesgo de transmisión de COVID-19, pero las distancias de dos metros podrían ser aún más efectivas. Asimismo, las máscaras faciales podrían proteger tanto a los trabajadores de la salud como al público en general contra la infección por SARS-CoV-2, y la protección ocular podría también proporcionar un beneficio adicional, aunque la certeza de la evidencia es baja para ambas formas de protección.

Estos hallazgos son los primeros en sintetizar la información directa sobre COVID-19, SARS y MERS, y proporcionar la mejor evidencia disponible hasta la actualidad sobre el uso óptimo de estas intervenciones comunes y simples para ayudar a aplanar la curva, que puede ser tomada en cuenta por los gobiernos y las autoridades sanitarias para dar consejos claros en entornos comunitarios y trabajadores de la salud. Sin embargo, es importante destacar que, incluso cuando se usan y combinan adecuadamente, ninguna de estas intervenciones ofrece protección completa contra la infección, y otras medidas de protección básicas (como la higiene de manos) son esenciales para reducir la transmisión del virus.

Como ya se ha desarrollado en otras revisiones ${ }^{4,5}$, para los trabajadores de la salud, el N95 y otras máscaras de tipo respirador podrían estar asociadas con una mayor protección contra la transmisión viral que las máscaras quirúrgicas o similares (por ejemplo, los barbijos reutilizables de algodón o gasa de 12 a 16 capas). Para la población general, este beneficio podría observarse incluso en entornos no relacionados con el cuidado de la salud, con máscaras desechables o de algodón reutilizable de 12 a 16 capas, aunque el uso masivo de máscaras faciales acarrea el riesgo de desviar los suministros de los trabajadores de la salud y otros cuidadores con mayor riesgo de infección a grupos de menor riesgo, lo que genera preocupación 6 .

A pesar de estos importantes hallazgos, la revisión resumida tiene algunas limitaciones, incluyendo que pocos estudios evaluaron el efecto de las intervenciones en entornos no relacionados con la atención médica, que la mayoría de las pruebas provienen de estudios de SARS y MERS; y que el efecto de la duración de la exposición sobre el riesgo de transmisión no se examinó específicamente.

Además, investigadores del Centro de Medicina Basada en la Evidencia (CEBM) de la Universidad de Oxford, tras realizar una exhaustiva revisión de la evidencia, incluyendo la resumida por esta revisión sistemática y meta-análisis, cuestionaron fuertemente sus resultados relativos al distanciamiento social ${ }^{7}$.

Incluso unos días antes de su publicación, en un comunicado, Carl Heneghan, director del CEBM, y Tom Jefferson, investigador honorario, realizaron un llamado de atención al detectar resultados no replicables para 13 de los 15 documentos analizados en la Figura 2 de la revisión de Chu et al, afirmando que "No hay evidencia científica que respalde la desastrosa regla de los dos metros. Se está utilizando investigación de mala calidad para justificar una política con enormes consecuencias para todos nosotros" 8,9 .

Los autores destacan que la evidencia para apoyar la regla de distanciamiento social de 2 metros para reducir la transmisión de COVID-19 consiste en estudios observacionales, retrospectivos, de baja calidad y con sesgos que socavan la fiabilidad de sus hallazgos. Coinciden en la necesidad de ensayos aleatorizados sólidos para informar mejor la evidencia de las intervenciones basadas en el distanciamiento social, y jerarquizan que el riesgo de contraer infecciones sería mayor en entornos de atención médica que en la comunidad, y mayor en espacios cerrados que en ambientes exteriores. También señalan que es necesario el lavado de manos y alentar el distanciamiento social, pero remarcan: lo que la evidencia no puede decir, es que exista una distancia "medida" que reduzca el riesgo de transmisión ${ }^{7}$.

No es la primera vez que la generación y el uso de la evidencia para enfrentar la pandemia de COVID-19 desatan controversias en la comunidad científica. En esta oportunidad, Henegan y Jefferson advierten sobre la tendencia a desarrollar, 
interpretar e informar las creencias anteriores, que puede llevar a distorsionar los métodos convencionales para crear una guía basada en la mejor evidencia disponible, y reflexionan sobre los riesgos del sesgo de confirmación, que deforma nuestro pensamiento y puede tener un efecto significativo en el funcionamiento adecuado de la sociedad al tergiversar la evidencia ${ }^{8,9}$.

\section{Conclusiones de la comentadora}

Esta investigación examina sistemáticamente la evidencia disponible sobre distanciamiento físico, máscaras faciales y protección ocular, para prevenir la transmisión de persona a persona del SARS-CoV-2 en los entornos comunitarios y de atención médica. Siempre que se interprete con cautela, teniendo en cuenta sus marcadas limitaciones, puede ser utilizada como guía para las recomendaciones de las autoridades sanitarias, mientras se genera la evidencia necesaria sobre tratamientos farmacológicos, vacunas y otras estrategias de protección personal.

Yanina Avendaño [ Servicio de Medicina Familiar y Comunitaria, Hospital Italiano de Buenos Aires. yanina.avendano@hospitalitaliano.org.ar ]

Avendaño Y. Distanciamiento físico óptimo, máscaras faciales y protección ocular para prevenir la transmisión de persona a persona del SARS-CoV-2. Evid Actual Pract Ambul. 2020;23(3):e002077. Comentado de: Chu DK et al. Physical distancing, face masks, and eye protection to prevent person transmission of SARS-CoV-2 and COVID-19: a systematic review and meta-analysis. The Lancet. 2020;395(10242):1973-1987. PMID: 32497510

\section{Referencias}

1. Chu DK, Akl EA, Duda S, et al. Physical distancing, face masks, and eye protection to prevent person-to-person transmission of SARS-CoV-2 and COVID-19: a systematic review and meta-analysis. The Lancet. 2020;Available from: 10.1016/S0140-6736(20)31142-9.

2. COVID-19 Dashboard by the Center for Systems Science and Engineering (CSSE) at Johns Hopkins University (JHU); 2020. Available from: https://coronavirus.jhu.edu/map.html [Last access: 2020-07-01].

3. Carrara C. Barbijos quirúrgicos versus máscaras N95 para la prevención de infecciones respiratorias virales en trabajadores de la salud. Comentado de: Bartoszko JJ, et al. Medical masks vs N95 respirators for preventing COVID-19 in healthcare workers: A systematic review and meta-analysis of randomized trials. Influenza Other Respir Viruses. 2020;00:1-9. Evid Actual PractAmbul. 2020;23(2):2067-2067. Available from: http://www. evidencia.org/index.php/Evidencia/article/view/6858.

4. Jefferson T, Mar CBD, Dooley L, et al. Physical interventions to interrupt or reduce the spread of respiratory viruses. Cochrane Database Syst Rev. 2011;(7):CD006207. Available from: 10.1002/14651858.CD006207.pub4.

5. Bartoszko JJ, Farooqi MAM, Alhazzani W, et al. Medical Masks vs N95 Respirators for Preventing COVID-19 in Healthcare Workers: A Systematic Review and Meta-Analysis of Randomized Trials. Influenza Other Respir Viruses. 2020;14(4):365-373. Available from: 10.1111/irv.12745.

6. World Health Organization. Interim Guidance. Rational use of personal protective equipment for coronavirus disease (COVID-19) and considerations during severe shortages; 2020. Available from: https://apps.who.int/iris/bitstream/handle/10665/331215/WHO-2019-nCov-IPCPPE_use-2020.1-eng. pdf [Last access: 2020-07-01].

7. Qureshi Z, Jones N, Temple R, et al.. What is the evidence to support the 2-metre social distancing rule to reduce COVID-19 transmission?; 2020. Available from: https://www.cebm.net/covid-19/what-is-the-evidence-to-support-the-2-metre-social-distancing-rule-to-reduce-covid19-transmission/ [Last access: 2020-07-01].

8. Heneghan C, Jefferson T. There is no scientific evidence to support the disastrous two-metre rule; 2020. Available from: https://www.telegraph.co. uk/news/2020/06/15/no-scientific-evidence-support-disastrous-two-metre-rule/.

9. Heneghan C, Jefferson T. COVID-19 Evidence is lacking for 2 meter distancing; 2020. Available from: https://www.cebm.net/covid-19/covid-19evidence-is-lacking-for-2-meter-distancing/ [Last access: 2020-07-01]. 